\title{
Aspirin effects on platelet gene expression are associated with a paradoxical, increase in platelet function.
}

\author{
Rachel Myers ${ }^{1}$, Thomas Ortel ${ }^{1}$, Sandeep Dave ${ }^{1}$, Alexander Waldrop ${ }^{1}$, Geoffrey Ginsburg ${ }^{1}$, \\ and Deepak Voora ${ }^{1}$ \\ ${ }^{1}$ Duke University
}

July 23, 2021

\begin{abstract}
Aspirin has known effects beyond inhibiting platelet cyclooxygenase-1 (COX1) that have been incompletely characterized. Transcriptomics can comprehensively characterize the on- and off-target effects of medications. We used a systems pharmacogenomics approach of aspirin exposure in volunteers coupled with serial platelet function and purified platelet mRNA sequencing to test the hypothesis that aspirin's effects on the platelet transcriptome are associated with platelet function. We prospectively recruited 74 adult volunteers for a randomized cross over study of $81-\mathrm{vs} .325 \mathrm{mg} /$ day, each for 4 weeks. Using mRNA sequencing of purified platelets collected before and after each 4-week exposure, we identified 208 aspirin-responsive genes with no evidence for dosage effects. In independent cohorts of healthy volunteers and patients with diabetes we validated aspirin's effects on five genes: EIF2S3, CHRNB1, EPAS1, SLC9A3R2, and HLA-DRA. Functional characterization of the effects of aspirin on mRNA as well as platelet ribosomal RNA demonstrated that aspirin may act as an inhibitor of protein synthesis. Database searches for small molecules that mimicked the effects of aspirin on platelet gene expression in vitro identified aspirin but no other molecules that share aspirin's known mechanisms of action. The effects of aspirin on platelet mRNA were correlated with higher levels of platelet function both at baseline and after aspirin exposure - an effect that counteracts aspirin's known antiplatelet effect. In summary, this work collectively demonstrates a dose-independent effect of aspirin on the platelet transcriptome that counteracts the well-known antiplatelet effects of aspirin.
\end{abstract}

Aspirin effects on platelet gene expression are associated with a paradoxical, increase in platelet function.

Rachel A Myers ${ }^{1}$, Thomas L Ortel ${ }^{2}$, Geoffrey S Ginsburg ${ }^{1}$, Alexander Waldrop ${ }^{3}$, Sandeep Dave ${ }^{3}$, and Deepak Voora $^{1}$

Affiliations : 1. Center for Applied Genomics \& Precision Medicine, Duke University School of Medicine, Durham NC; 2. Division of Hematology, Department of Medicine, Duke University Medical Center, Durham NC; 3. Center for Genomics and Computational Biology, Duke University, Durham NC

Financial disclosures : None

Funding : This work was supported by grant funding from the National Institutes of Health (R01HL118049).

Keywords : aspirin, platelet aggregometry, transcriptomics, cardiovascular disease

Abstract

Aspirin has known effects beyond inhibiting platelet cyclooxygenase-1 (COX1) that have been incompletely characterized. Transcriptomics can comprehensively characterize the on- and off-target effects of medications. We used a systems pharmacogenomics approach of aspirin exposure in volunteers coupled with serial platelet function and purified platelet mRNA sequencing to test the hypothesis that aspirin's effects on the platelet 
transcriptome are associated with platelet function. We prospectively recruited 74 adult volunteers for a randomized cross over study of 81- vs. $325 \mathrm{mg} /$ day, each for 4 weeks. Using mRNA sequencing of purified platelets collected before and after each 4-week exposure, we identified 208 aspirin-responsive genes with no evidence for dosage effects. In independent cohorts of healthy volunteers and patients with diabetes we validated aspirin's effects on five genes: EIF2S3, CHRNB1, EPAS1, SLC9A3R2, and HLA-DRA. Functional characterization of the effects of aspirin on mRNA as well as platelet ribosomal RNA demonstrated that aspirin may act as an inhibitor of protein synthesis. Database searches for small molecules that mimicked the effects of aspirin on platelet gene expression in vitro identified aspirin but no other molecules that share aspirin's known mechanisms of action. The effects of aspirin on platelet mRNA were correlated with higher levels of platelet function both at baseline and after aspirin exposure - an effect that counteracts aspirin's known antiplatelet effect. In summary, this work collectively demonstrates a dose-independent effect of aspirin on the platelet transcriptome that counteracts the well-known antiplatelet effects of aspirin.

\section{Introduction}

Aspirin use is recommended by the United States Preventive Services Task Force to prevent cardiovascular disease (CVD) in selected, high risk patients.[1] It is well known that aspirin inhibits platelet cyclooxygenase 1 (COX1)[2]; however, aspirin has additional effects on platelets, which are not fully explained by its effects on COX-1. For example, $81 \mathrm{mg} /$ day aspirin is sufficient to fully suppress platelet COX1[3] yet higher doses cause additional reductions in platelet function as assessed by non-COX1 dependent platelet function assays such as adenosine diphosphate (ADP) and collagen induced platelet aggregation.[4] In addition, despite consistent platelet COX-1 inhibition, non-COX1 dependent measures of platelet function wane over time[5] suggesting some adaptation of platelets to chronic aspirin exposure. Residual platelet function measured using non-COX1 dependent platelet function assays are risk markers for future cardiovascular events during aspirin therapy. [6, 7] Last after decades of uncertainty around the appropriate aspirin dose for cardiovascular risk reduction[8] only recently has there been randomized trial data comparing the effectiveness and safety of low- vs. high-dose aspirin. This trial did not identify major differences in efficacy or safety between aspirin doses; however, nearly $50 \%$ of patients crossed over to the alternative dose with 3 weeks of randomization. [9] Therefore, gaps in our knowledge around one of the most prescribed medicines worldwide remain.

Although anucleate, it is well known that platelets are a rich source of messenger RNA (mRNA). Platelets are invested with mRNA from megakaryocytes during thrombopoiesis in a regulated manner[10], can uptake transcripts from other vascular cells[11], and are also a major source of transcripts found in plasma extracellular vesicles [12]. Genetic and gene expression studies have confirmed that variable platelet mRNA levels lead to variable levels of platelet function.[13-15] We have previously shown that a blood-based, "aspirin response signature" of transcripts primarily of platelet origin correlate with platelet function on aspirin and predict future cardiovascular events in patients taking aspirin.[16] These data support the concept that the biologic basis for variable platelet function lies, at least in part, in variable platelet gene expression. We[17] and others[18] have shown that aspirin (or its stable metabolite salicylate) can alter expression of selected genes in megakaryocytes as well as in circulating platelets in patients exposed to aspirin. However, these prior studies were limited in scope as they did not systematically characterize aspirin's effects on platelet gene expression.

Based on these knowledge gaps, we pursued the hypothesis that variability in the platelet function response to aspirin is associated with aspirin induced changes in the platelet transcriptome. We tested this hypothesis using an open label, randomized crossover study of low- vs. high-dose aspirin in human volunteers with longitudinal COX-1 direct and indirect assays of platelet function, drug concentrations, and RNA profiling at each time point.

\section{Methods}

Human subject protocols

Discovery Cohort 
We conducted a prospective, open label, randomized cross-over study to assess the effects of antiplatelet therapy (non-enteric coated aspirin $81 \mathrm{mg} /$ day, aspirin $325 \mathrm{mg} /$ day, and ticagrelor $90 \mathrm{mg}$ twice daily) on platelet function and gene expression as previously described.[19] The results of aspirin exposure are presented here. The Supplemental Methods provides more details of inclusion/exclusion criteria. The Duke Institutional Review Board approved the study protocol.

Validation cohorts

Banked, platelet RNA and platelet function data from previously described[20] cohorts of healthy volunteers or patients with diabetes exposed to $325 \mathrm{mg}$ /day aspirin for 2 or 4 weeks was used for independent validation.

\section{Platelet function and salicylate testing}

Phlebotomy for platelet function testing was performed as previously described with additional details in the Supplemental Methods.[20]

Platelet RNA purification

Platelet isolation from human blood using a previously described[21] leukocyte depletion (LD) procedure is briefly described here with additional details in Supplemental Methods.

\section{Platelet RNA isolation}

Platelet RNA samples from the same participant were batched together at every stage from platelet RNA extraction through sequencing in order to minimize introducing technical artifacts between visits.

RNA Isolation from purified platelets using a phenol-chloroform method as described in the Supplemental Methods.

Platelet RNA quality analysis and sequencing

Total RNA quality and quantity was assessed using the Agilent Bioanalyzer 2100 and the Qubit fluorometer, respectively. Samples with sufficient quantity and quality RNA went on to poly(A) mRNA capture and construction of stranded mRNA-seq libraries from total RNA was achieved using the commercially available KAPA Stranded mRNA-Seq Kit (catalog \#KK8421) using the manufacturer's protocol and as described in the Supplemental Methods.

Platelet mRNA sequencing alignment and expression quantification

See Supplemental Methods for details regarding quantification of platelet mRNA transcripts.

Nanostring expression profiling

Targeted gene expression of selected transcripts identified using RNAseq was performed in the validation cohort using Nanostring technology as described in the Supplemental Methods.[22]

Data access

All platelet function and RNAsequencing data used in this manuscript is available through Gene Expression Omnibus (GSE158765)

\section{Statistical analyses}

See Supplemental Methods for details of statistical methods used to analyze platelet function and gene expression data.

Results

Aspirin's effects on platelet function outcomes

Eighty-six participants completed a baseline visit, 81 exposed to low-dose aspirin, and 79 exposed to highdose (Figure 1A). To assess the extent to which platelet COX-1 was inhibited we used serum thromboxane 
B2 (TXB2), and arachidonic acid (AA) induced platelet aggregation. After 4 weeks of daily dosing, both $81 \mathrm{mg}$ and $325 \mathrm{mg}$ equivalently suppressed platelet COX-1 as assessed by (AA) induced platelet aggregation (Figure 1B) and TXB2 (Figure 1B). To assess non-COX1 dependent assays of platelet function, we used a previously described platelet function score (PFS, see Supplemental Methods) that aggregates nine different, non-COX1 dependent assays of platelet function into a single score. Higher PFS values reflect greater platelet function. After 4 -weeks of daily aspirin, both 81 and 325mg doses reduced PFS $(\mathrm{p}<0.001)$ with slightly greater reduction with $325 \mathrm{mg}$ vs. $81 \mathrm{mg}(\mathrm{p}=0.07)$ (Figure 1D). Therefore, daily doses of low- and high dose aspirin reduce COX-1 and non COX-1 measures of platelet function. Compared to low-dose aspirin, high dose aspirin produces equivalent COX-1 suppression and slightly greater non-COX 1 inhibition of platelet function.

\section{Aspirin's effects of platelet gene expression}

After quality analysis of the platelet RNA sequencing data there were 312 platelet mRNA datasets available for analysis from 73 unique patients representing 14333 transcripts. In general, the level of contamination by granulocytes was low (proportion of samples withPTPRC /ITGA2B count ratio [?] $0.01=6 / 312$ ) and did not vary by treatment (likelihood ratio test $\mathrm{P}$-value $=0.11$ ). We have previously shown that platelet gene expression levels are highly correlated within an individual between Visits 1 and Visit 4.[23] Therefore in all subsequent analyses, these two visits were treated equivalently as no-drug treatment visits and no adjustment for leukocyte contamination was performed.

To test our primary hypothesis that aspirin exposure alters the platelet mRNA transcriptome, we used a mixed-effects regression framework to identify transcripts that changed with any aspirin exposure compared to no drug treatment visits. In a univariate analysis using the false discovery rate (FDR) q-value $<0.10$ to account for multiple testing we identified 208 transcripts that were differentially expressed (43 down regulated, 165 upregulated, Figure 2, Supplemental Data File 1) after aspirin exposure. To test for aspirindose response relationships we used two complementary approaches: 1) contrasting the effects of $81 \mathrm{mg}$ vs. $325 \mathrm{mg}$ /day and 2) correlating gene expression with salicylate concentrations during aspirin treatment. We found no evidence for differences on platelet gene expression for the set of 208 gene aspirin differentially expressed genes and higher aspirin dose or salicylate concentrations (enrichment p-values $=0.3$ and 0.9 , respectively).

We used banked platelet RNA from prior research cohorts[19] of healthy adults and patients with diabetes $(\mathrm{n}=91$ across both cohorts) exposed to $81 \mathrm{mg} /$ day or $325 \mathrm{mg}$ /day aspirin using a custom Nanostring assay to validate the effects of aspirin. Due to limits on the number of transcripts available in a Nanostring assay, we prioritized the list 208 transcripts based on mean expression and robustness to various starting points for quantifying expression, and genes that exhibited positive correlation $(\mathrm{r}>0.25)$ between RNAseq and Nanostring to a subset of 51 genes to test for validation. Among these 51 genes, we found five that independently validated in the replication cohorts (fixed effects meta-analysis p-value for aspirin effect $<$ 0.05) as changing in response to aspirin exposure: EIF2S3, CHRNB1, EPAS1, SLC9A3R2, and HLA-DRA . (Figure 2B)

Functional characterization of aspirin effects on platelet gene expression:

To characterize the functional effects of aspirin on platelet gene expression we used two complementary approaches: 1) Gene Set Enrichment Analyses (GSEA) which uses all transcripts from the mRNA sequencing experiment and 2) Connectivity Map (CMap) using a broader list (FDR $<15 \%$ ) of differentially expressed genes identified through mRNA sequencing.

Using GSEA, we identified gene sets that were enriched (GSEA family wise error rate $[\mathrm{FWER}] \mathrm{p}<0.05$ ) for genes that were upregulated by aspirin exposure. Many of these gene sets were related to ribosome biogenesis (Figure 3B) full list available in Supplementary File 2. Gene sets that were enriched in genes that were downregulated in response to aspirin reflected calcium/mTOR signaling, platelet aggregation, glucose metabolism, and cytoskeletal pathways (Figure 3A and Supplementary File 2). Therefore, the global effects of aspirin on the platelet transcriptome identified effects on multiple pathways including many related to 
protein synthesis.

CMap searches for similarities in the effect of aspirin on platelets using mRNA sequencing to the effects of 2837 small molecules on gene expression across nine cell lines. In this analysis, molecules with positive 'tau' scores (range -100 to +100) are interpreted as producing similar effects in cell lines as aspirin exposure in platelets and negative tau scores reflecting effects of aspirin in cells that are in the opposite direction as those found in platelets. The top molecules to emerge from this analysis $(|\operatorname{tau}|>90)$ are listed in Figure 3B. In addition to identifying aspirin $($ tau $=94)$, five additional molecules not known to share mechanisms with aspirin were identified: inhibitors of protein synthesis (cephaeline and emetine), protein kinase A (H7), protein kinase C (GSK-3-inhibitor II), and tubulin (parbendazole). In contrast, none of the 121 Cmap molecules that share known mechanisms of action with aspirin (COX1, COX2, NFKB, or TBXAS1 inhibitors or AMPK activators) produced tau scores as large as those as aspirin. (Figure 3B). Therefore, in cellular models, we validate the global effects of aspirin on gene expression and raise the hypothesis of potential non-canonical effects of aspirin, including a role in inhibiting protein synthesis.

Among the pathways identified, we chose to focus on aspirin's effects on protein synthesis given that EIF2S3 (Eukaryotic Translation Initiation Factor 2 Subunit Gamma) validated as an aspirin-responsive gene in our human platelet data (Figure 2B), that ribosomal biosynthesis emerged from GSEA analyses (Figure 3A), and the identification of more than 1 inhibitor of protein synthesis from CMap analyses (Figure 3B). We explored the extent to which that aspirin exposure results in altered platelet ribosomal RNA (rRNA) levels which are well-known to regulate global protein synthesis.[24] Using available, banked platelet RNA samples from healthy subjects, we measured 18S rRNA levels after 4 and 8 weeks of $325 \mathrm{mg}$ /day aspirin exposure using qPCR. Aspirin exposure of $325 \mathrm{mg} /$ day for at least 4 weeks was associated $(\mathrm{p}=0.04)$ with lower platelet $18 \mathrm{~S}$ rRNA levels with a trend towards greater effects for longer durations of aspirin exposure. (Figure 3C)

\section{Effects of aspirin on platelet gene expression and platelet function outcomes}

To test the hypothesis that aspirin's effects on platelet mRNA correlates with aspirin's effects on non-COX1 dependent platelet function we first summarized the overall behavior of the 51 , prioritized, differentially expressed genes identified in the Discovery cohort into a single AES score (see Methods). By definition, the AES score increases in response to aspirin exposure with no dosage effects (Figure 4A). We used the aggregate AES to correlate with platelet function scores across all cohorts (Discovery and both Validation) to test for the relationship between the effects of aspirin on gene expression and platelet function.

At baseline, prior to aspirin exposure, platelets with higher AES scores can be thought of as having an endogenous "aspirin-like" effect in their platelet transcriptomes. Prior to aspirin exposure, higher AES scores correlated with higher PFS both before (effect $=0.49,95 \%$ confidence interval $[\mathrm{CI}]: 0.10-0.89$, random effect model p-value $=0.01$, Figure $4 \mathrm{~B}$ and $4 \mathrm{D}$ ) with higher levels of platelet function. In aspirin treated samples, higher AES levels reflect those participants in whom aspirin exposure resulted in a higher AES levels (due to higher baseline or change upon aspirin exposure). After aspirin exposure, higher AES levels were similarly correlated with higher platelet function (effect $=0.70,95 \%$ confidence interval $[\mathrm{CI}]: 0.30-1.18$, random effect model p-value $=0.002$, Figure $4 \mathrm{C}$ and $4 \mathrm{E}$ ). Therefore, the biological pathways impacted by aspirin and quantified through platelet gene expression profiling indicate that aspirin produces a paradoxical effect that increases platelet aggregation ex vivo . Higher aspirin-like effects (i.e., higher AES scores) either at baseline or in response to aspirin exposure, in aggregate, correlate with higher PFS scores, an effect that is opposite in direction with aspirin's known effects on inhibiting platelet COX-1 and platelet function.

\section{Discussion}

Aspirin is widely used to prevent cardiovascular disease and colorectal cancers. While its role in as an antiplatelet agent is well described, there remain large gaps in our knowledge of its diverse effects or optimal dose beyond cardiovascular disease. In this prospective randomized study of different aspirin doses, we aimed to comprehensively study the effects of aspirin on platelet gene expression and function. We tested the hypothesis that aspirin's effects on platelet gene expression contribute to variability in platelet function in response to aspirin exposure. Compared to daily low-dose aspirin high-dose aspirin exhibited equivalent 
inhibition of platelet COX1 and slightly greater platelet inhibition in non-COX-1 dependent measures of platelet function. We found that aspirin exposure results in a mild perturbation in platelet mRNA ( $1.5 \%$ of transcripts affected) with no evidence for dose effects. Using multiple, complimentary lines of evidence, we found that aspirin exposure appears to have effects as an inhibitor of protein synthesis in platelets and/or megakaryocytes. In terms of platelet function, aspirin's effects on platelet gene expression appear to counteract aspirin's platelet inhibitory effects on platelet COX-1. In summary, using a diverse array of platelet function and molecular tools, there appear to be effects of aspirin beyond platelet COX-1 inhibition that are dose-independent and counteract the main antiplatelet effect of aspirin.

Aspirin is well known to inhibit platelet COX-1[2] which is critical for arachidonic acid-induced platelet aggregation. Additionally, aspirin has been attributed with a wide array of additional mechanisms of action including: NFKB/IKB inhibition[25], AMPK activation[26], COX2 inhibition[27], RUNX1 pathway modulation[28]. It is unknown the extent to which these off-target effects of aspirin contribute to its clinical effects in preventing cardiovascular disease or if they are relevant in typical prescribed doses $(<325 \mathrm{mg} /$ day $)$. Many of these effects are attributed to the main metabolite of aspirin, salicylate, which has a longer half-life than aspirin and achieves systemic levels in the 30-100 $\mu \mathrm{M}[29]$ range with typical daily dosing. Alternatively, they may arise from downstream effects of COX-1 inhibition on arachidonic acid metabolites. Aspirin and salicylates have previously been shown to inhibit protein synthesis in vitro [30,31] and in vivo [31] in the gastrointestinal tract of patients taking $600 \mathrm{mg}$ /day. This effect appears to be mediated through activation of AMPK and mTOR pathway[31] and acetylation of non-COX1 targets [32]. Our findings of aspirin potentially acting as an inhibitor of protein synthesis are in line with these previous observations and extend the findings to platelets from humans taking lower, therapeutic aspirin doses. Among the top genes that independently validated as being modulated by aspirin exposure were EIF2S3which encodes for a core subunit of eukaryotic translation initiation factor-2 (eIF2) complex, which is essential for protein synthesis.[33] The suppression of aspirin on $18 \mathrm{~S}$ platelet ribosomal RNA subunit (which is not captured in RNA sequencing) in platelets in our study further supports this role for inhibition of protein synthesis in platelets. Our finding that aspirin's effects in platelets is recapitulated in cellular data suggest a potential direct role of aspirin (or its metabolite) modifying gene expression likely in megakaryocytes. We found no significant effect of aspirin dose on aspirin induced changes in platelet gene expression. Therefore, at $81 \mathrm{mg} /$ day, any effects on platelet gene expression appear to be saturated. Although our study was not designed to identify the mechanism of action of the observed effects, our analysis of the CMap data (Figure 3B) identified aspirin but no other COX-1, COX-2, or thromboxane inhibitors as causing similar changes in gene expression that we observed in human platelets. Therefore, these findings raise the hypothesis of a non-canonical effect of aspirin on protein synthesis in human megakaryocytes and/or platelets.

The primary target of aspirin is acetylation of platelet COX-1 which is uniformly suppressed when adherence and absorption are adequately addressed.[3] In our studies, we show that a single 325mg dose of nonenteric coated aspirin suppresses COX1 and that after multiple daily doses, similar suppression is achieved with $81 \mathrm{mg}$ /day. However, platelet COX-1 variably contributes to collagen, ADP, and epinephrine induced aggregation such that in the setting of complete COX-1 inhibition there is residual platelet aggregation using these agonists. This residual aggregation is referred to as "non-COX-1 dependent" [5] or "COX-1 indirect" [34] in the literature. We have previously described an approach to aggregate multiple measures of non-COX-1 dependent platelet function using a platelet function score (PFS).[5] In our current study, we found that high dose aspirin may result in further reduction in PFS (Figure 1D) despite complete suppression of platelet COX1 (Figure 1C), a finding that is consistent with the prior ASPECT[4] trial that found greater platelet inhibitory effects of higher aspirin dose on non COX-1 dependent platelet function pathways. The extent to which this greater, non-COX1 mediated platelet inhibition of high-dose aspirin on cardiovascular events (or bleeding) is unknown but will be clarified by the ongoing ADAPTABLE clinical trial comparing low vs. high dose aspirin (NCT02697916). Until then, our findings suggest that there are no large differences between $325 \mathrm{mg} /$ day and $81 \mathrm{mg}$ /day aspirin and from the perspective of platelet function and gene expression there is no clear advantage to the higher dosage.

To examine aspirin effects on platelet gene expression with platelet function, we used an aspirin exposure 
signature which aggregates the expression of aspirin responsive transcripts into a single "score" with higher scores representing a greater aspirin effect. This approach allowed us to quantify the biological effects of aspirin using gene expression and also assign an "aspirin-like" effect to all samples used in this study before and after aspirin exposure. Using this score, we found that the effect of aspirin on platelet gene expression appears to attenuate the platelet inhibitory effects of aspirin on COX-1 inhibition. The overall magnitude of this effect is relatively small, however. The average effect of aspirin on increasing the AES score was 0.50 (Figure $4 \mathrm{~A}$ ) and from the meta-analysis, this magnitude of effect translates into 0.43 higher PFS units on aspirin (Figure 4E). The magnitude of aspirin's effects of inhibiting platelet COX-1 is approximately 4.0 PFS units based on the change in PFS 3 hours after a single 325mg aspirin effect of aspirin on inhibiting platelet COX-1 (Figure 1D). Therefore, during a 4-week aspirin exposure the effects of aspirin on platelet gene expression are relatively minor (10\% of overall magnitude) compared to the magnitude of effect on inhibiting platelet COX-1. The apparent time-dependent effects of aspirin on platelet ribosomal RNA (Figure 3C) suggest that longer durations of aspirin may be associated with larger effects on platelet gene expression. In fact, studies of patients taking aspirin for more than 2 months showed greater changes in platelet gene expression and higher residual, non-COX1 platelet function.[18] Therefore, future work in patients chronically exposed to aspirin may identify a greater contribution of aspirin's effects on platelet gene expression, high residual platelet reactivity, and risk for cardiovascular events.

In conclusion, using a systems pharmacogenomics approach to studying the effects of aspirin in a rigorous, human experimental model of drug response, unbiased, genome-wide gene expression analysis, independent validation, and correlation with ex vivo measures of platelet function we identify novel effects of aspirin on platelets that counteract its canonical, pharmacological effects as a platelet inhibitor that may be part of what has been previously described as an adaptive response to exposure to antiplatelet therapy.[35] We extend prior observations of this effect of aspirin to platelets from humans exposed $81 \mathrm{mg} /$ day of aspirin with no evidence for dose-response. Overall, this effect appears to attenuate the well-described inhibitory effects of aspirin on inhibiting platelet COX-1. The extent to which these effects on platelet gene expression impact the clinical effect of aspirin on preventing CVD are currently not known but deserve future consideration.

\section{Highlights}

- What is the current knowledge on the topic?

- Aspirin is widely prescribed to prevent cardiovascular disease through inhibition of platelet COX1. Aspirin is known to have effects beyond inhibiting platelet COX-1; however, characterization of non-COX1 effects in platelets has been lacking.

- What question did this study address?

- This study comprehensively characterized the on- and off-target effects of aspirin on platelet gene expression and platelet function.

- What does this study add to our knowledge?

- Using an experimental protocol of human aspirin exposure and serial platelet transcriptomic and functional testing, we identified a non-canonical effect of aspirin on inhibiting protein synthesis pathways in platelets. This effect acts to attenuate the platelet inhibitory effects of aspirin on platelet COX-1

- How might this change clinical pharmacology or translational science?

- Compared to low-dose aspirin we found no major differences with high-dose aspirin with respect to platelet function or platelet transcriptome and thus no rationale for using high-dose aspirin. Patients chronically prescribed aspirin to prevent cardiovascular disease that have an accentuated platelet transcriptional response may experience smaller than expected reductions in cardiovascular risk.

\section{References}

1. USPTF. Aspirin to Prevent Cardiovascular Disease and Cancer. In, 2015.

2. Roth GJ, Majerus PW. The mechanism of the effect of aspirin on human platelets. I. Acetylation of a particulate fraction protein. J Clin Invest 1975; 56: 624-32.

3. Grosser T, Fries S, Lawson JA, Kapoor SC, Grant GR, FitzGerald GA. Drug Resistance and Pseudore- 
sistance: An Unintended Consequence of Enteric Coating Aspirin. Circulation 2012.

4. Gurbel PA, Bliden KP, DiChiara J, Newcomer J, Weng W, Neerchal NK, Gesheff T, Chaganti SK, Etherington A, Tantry US. Evaluation of dose-related effects of aspirin on platelet function: results from the Aspirin-Induced Platelet Effect (ASPECT) study. Circulation 2007; 115: 3156-64.

5. Voora D, Ortel TL, Lucas JE, Chi JT, Becker RC, Ginsburg GS. Time-dependent changes in non-COX1-dependent platelet function with daily aspirin therapy. Journal of Thrombosis and Thrombolysis 2012; 33: 246-57.

6. Frelinger AL, Li Y, Linden MD, Barnard MR, Fox ML, Christie DJ, Furman MI, Michelson AD. Association of Cyclooxygenase-1-Dependent and -Independent Platelet Function Assays With Adverse Clinical Outcomes in Aspirin-Treated Patients Presenting for Cardiac Catheterization. Circulation 2009; 120: 258696.

7. Qayyum R, Becker DM, Yanek LR, Faraday N, Vaidya D, Mathias R, Kral BG, Becker LC. Greater collagen-induced platelet aggregation following cyclooxygenase 1 inhibition predicts incident acute coronary syndromes. Clin Transl Sci 2015; 8: 17-22.

8. Johnston A, Jones WS, Hernandez AF. The ADAPTABLE Trial and Aspirin Dosing in Secondary Prevention for Patients with Coronary Artery Disease. Curr Cardiol Rep 2016; 18 : 81.

9. Jones WS, Mulder H, Wruck LM, Pencina MJ, Kripalani S, Muñoz D, Crenshaw DL, Effron MB, Re RN, Gupta K, Anderson RD, Pepine CJ, Handberg EM, Manning BR, Jain SK, Girotra S, Riley D, DeWalt DA, Whittle J, Goldberg YH, Roger VL, Hess R, Benziger CP, Farrehi P, Zhou L, Ford DE, Haynes K, VanWormer JJ, Knowlton KU, Kraschnewski JL, Polonsky TS, Fintel DJ, Ahmad FS, McClay JC, Campbell JR, Bell DS, Fonarow GC, Bradley SM, Paranjape A, Roe MT, Robertson HR, Curtis LH, Sharlow AG, Berdan LG, Hammill BG, Harris DF, Qualls LG, Marquis-Gravel G, Modrow MF, Marcus GM, Carton TW, Nauman E, Waitman LR, Kho AN, Shenkman EA, McTigue KM, Kaushal R, Masoudi FA, Antman EM, Davidson DR, Edgley K, Merritt JG, Brown LS, Zemon DN, McCormick TE, Alikhaani JD, Gregoire KC, Rothman RL, Harrington RA, Hernandez AF. Comparative Effectiveness of Aspirin Dosing in Cardiovascular Disease. New England Journal of Medicine 2021; 384: 1981-90.

10. Cecchetti L, Tolley ND, Michetti N, Bury L, Weyrich AS, Gresele P. Megakaryocytes differentially sort mRNAs for matrix metalloproteinases and their inhibitors into platelets: a mechanism for regulating synthetic events. Blood 2011; 118: 1903-11.

11. Clancy L, Beaulieu LM, Tanriverdi K, Freedman JE. The role of RNA uptake in platelet heterogeneity. Thromb Haemost 2017; 117: 948-61.

12. Clancy L, Freedman JE. Blood-Derived Extracellular RNA and Platelet Pathobiology: Adding Pieces to a Complex Circulating Puzzle. Circ Res 2016; 118: 374-6.

13. Faraday N, Yanek LR, Yang XP, Mathias R, Herrera-Galeano JE, Suktitipat B, Qayyum R, Johnson AD, Chen M-H, Tofler GH, Ruczinski I, Friedman AD, Gylfason A, Thorsteinsdottir U, Bray PF, O'Donnell CJ, Becker DM, Becker LC. Identification of a specific intronic PEAR1 gene variant associated with greater platelet aggregability and protein expression. Blood 2011; 118: 3367-75.

14. Goodall AH, Burns P, Salles I, Macaulay IC, Jones CI, Ardissino D, de Bono B, Bray SL, Deckmyn H, Dudbridge F, Fitzgerald DJ, Garner SF, Gusnanto A, Koch K, Langford C, O'Connor MN, Rice CM, Stemple D, Stephens J, Trip MD, Zwaginga JJ, Samani NJ, Watkins NA, Maguire PB, Ouwehand WH. Transcription profiling in human platelets reveals LRRFIP1 as a novel protein regulating platelet function. Blood 2010; 116: 4646-56.

15. Nagalla S, Shaw C, Kong X, Kondkar AA, Edelstein LC, Ma L, Chen J, McKnight GS, Lopez JA, Yang L, Jin Y, Bray MS, Leal SM, Dong JF, Bray PF. Platelet microRNA-mRNA coexpression profiles correlate with platelet reactivity. Blood 2011; 117: 5189-97. 
16. Voora D, Cyr D, Lucas J, Chi J-T, Dungan J, McCaffrey TA, Katz R, Newby LK, Kraus WE, Becker RC, Ortel TL, Ginsburg GS. Aspirin Exposure Reveals Novel Genes Associated With Platelet Function and Cardiovascular Events. Journal of the American College of Cardiology 2013; 62: 1267-76.

17. Voora D, Rao AK, Jalagadugula GS, Myers R, Harris E, Ortel TL, Ginsburg GS. Systems Pharmacogenomics Finds RUNX1 Is an Aspirin-Responsive Transcription Factor Linked to Cardiovascular Disease and Colon Cancer. EBioMedicine 2016.

18. Massimi I, Lotti LV, Temperilli F, Mancone M, Sardella G, Calcagno S, Turriziani O, Frati L, Pulcinelli FM. Enhanced platelet MRP4 expression and correlation with platelet function in patients under chronic aspirin treatment. Thrombosis and Haemostasis 2016.

19. Friede KA, Infeld MM, Tan RS, Knickerbocker HJ, Myers RA, Dubois LG, Thompson JW, KaddurahDaouk R, Ginsburg GS, Ortel TL, Voora D. Influence of Sex on Platelet Reactivity in Response to Aspirin. J Am Heart Assoc 2020; 9: e014726.

20. Voora D, Cyr D, Lucas J, Chi JT, Dungan J, McCaffrey TA, Katz R, Newby LK, Kraus WE, Becker RC, Ortel TL, Ginsburg GS. Aspirin Exposure Reveals Novel Genes Associated with Platelet Function and Cardiovascular Events. J Am Coll Cardiol 2013.

21. Kondkar AA, Bray MS, Leal SM, Nagalla S, Liu DJ, Jin Y, Dong JF, Ren Q, Whiteheart SW, Shaw C, Bray PF. VAMP8/Endobrevin is over expressed in hyperreactive human platelets: suggested role for platelet micro-RNA. J Thromb Haemost 2009.

22. Geiss GK, Bumgarner RE, Birditt B, Dahl T, Dowidar N, Dunaway DL, Fell HP, Ferree S, George RD, Grogan T, James JJ, Maysuria M, Mitton JD, Oliveri P, Osborn JL, Peng T, Ratcliffe AL, Webster PJ, Davidson EH, Hood L, Dimitrov K. Direct multiplexed measurement of gene expression with color-coded probe pairs. Nat Biotechnol 2008; 26: 317-25.

23. Rondina MT, Voora D, Simon LM, Schwertz H, Harper JF, Lee O, Bhatlekar SC, Li Q, Eustes AS, Montenont E, Campbell RA, Tolley ND, Kosaka Y, Weyrich AS, Bray PF, Rowley JW. Longitudinal RNASeq Analysis of the Repeatability of Gene Expression and Splicing in Human Platelets Identifies a Platelet SELP Splice QTL. Circ Res 2020; 126: 501-16.

24. Mills EW, Green R. Ribosomopathies: There's strength in numbers. Science 2017; 358: eaan2755.

25. Kopp E, Ghosh S. Inhibition of NF-kappa B by sodium salicylate and aspirin. Science 1994; 265: 956-9.

26. Hawley SA, Fullerton MD, Ross FA, Schertzer JD, Chevtzoff C, Walker KJ, Peggie MW, Zibrova D, Green KA, Mustard KJ, Kemp BE, Sakamoto K, Steinberg GR, Hardie DG. The ancient drug salicylate directly activates AMP-activated protein kinase. Science 2012; 336: 918-22.

27. Mitchell JA, Akarasereenont P, Thiemermann C, Flower RJ, Vane JR. Selectivity of nonsteroidal antiinflammatory drugs as inhibitors of constitutive and inducible cyclooxygenase. Proceedings of the National Academy of Sciences 1993; 90: 11693-97.

28. Voora D, Rao AK, Jalagadugula GS, Myers R, Harris E, Ortel TL, Ginsburg GS. Systems Pharmacogenomics Finds RUNX1 Is an Aspirin-Responsive Transcription Factor Linked to Cardiovascular Disease and Colon Cancer. EBioMedicine 2016; 11: 157-64.

29. Nagelschmitz J, Blunck M, Kraetzschmar J, Ludwig M, Wensing G, Hohlfeld T. Pharmacokinetics and pharmacodynamics of acetylsalicylic acid after intravenous and oral administration to healthy volunteers. Clin Pharmacol 2014; 6: 51-59.

30. Silva AM, Wang D, Komar AA, Castilho BA, Williams BRG. Salicylates Trigger Protein Synthesis Inhibition in a Protein Kinase R-like Endoplasmic Reticulum Kinase-dependent Manner. Journal of Biological Chemistry 2007; 282: 10164-71. 
31. Din FVN, Valanciute A, Houde VP, Zibrova D, Green KA, Sakamoto K, Alessi DR, Dunlop MG. Aspirin inhibits mTOR signaling, activates AMP-activated protein kinase, and induces autophagy in colorectal cancer cells. Gastroenterology 2012; 142: 1504-15.e3.

32. Wang J, Zhang C-J, Zhang J, He Y, Lee YM, Chen S, Lim TK, Ng S, Shen H-M, Lin Q. Mapping sites of aspirin-induced acetylations in live cells by quantitative acid-cleavable activity-based protein profiling (QA-ABPP). Scientific Reports 2015; 5: 7896.

33. Moortgat S, Désir J, Benoit V, Boulanger S, Pendeville H, Nassogne MC, Lederer D, Maystadt I. Two novel EIF2S3 mutations associated with syndromic intellectual disability with severe microcephaly, growth retardation, and epilepsy. Am J Med Genet A 2016; 170: 2927-33.

34. Becker DM, Segal J, Vaidya D, Yanek LR, Herrera-Galeano JE, Bray PF, Moy TF, Becker LC, Faraday N. Sex differences in platelet reactivity and response to low-dose aspirin therapy. Jama 2006; 295: 1420-7.

35. Lowenstern A, Storey RF, Neely M, Sun JL, Angiolillo DJ, Cannon CP, Himmelmann A, Huber K, James SK, Katus HA, Morais J, Siegbahn A, Steg PG, Wallentin L, Becker RC, Investigators P. Platelet-related biomarkers and their response to inhibition with aspirin and p2y12-receptor antagonists in patients with acute coronary syndrome. J Thromb Thrombolysis 2017; 44: 145-53.

\section{Figure Legends}

Figure 1 Aspirin exposure study design and effects on platelet function. Panel A: Sequence of drug exposures and washout periods for volunteers enrolled in the discovery cohort. Panel B: Serum thromboxane B2 (TXB2, y-axis) concentrations $(\mathrm{ng} / \mathrm{mL}$ ) at baseline and after 4 weeks of $81 \mathrm{md} /$ day and 325mg/day aspirin exposure. Panel C: Maximum light transmittance aggregometry percent aggregation (y-axis) induced by arachidonic acid (AA) before and after 3-hours or 4-weeks of daily aspirin exposure (81- and $325 \mathrm{mg} /$ day). Panel D: An aggregate platelet function score (PFS, y-axis, see Methods) of non-COX1 dependent platelet function measures measured before and 3-hours and 4-weeks after daily aspirin exposure. Box-whisker plots indicate median (bold line), interquartile range (box), and outliers. Values connecting boxes indicate p-values for comparison between exposures.

Figure 2 Discovery and validation of aspirin responsive transcripts. Panel A: "Volcano" plot of genes detected by messenger RNA sequencing and tested for association with aspirin exposure. Each point represents a gene of interest, its fold change with aspirin exposure $(\log 2, \mathrm{x}$-axis $)$, and p-value $(-\log 10, \mathrm{y}-$ axis). Colored points represent those that were significant after adjustment for multiple comparisons (false discovery rate $<0.1$ ). Panel B: "Forest" plots of meta-analysis for each independently validated aspirin responsive gene $(\mathrm{n}=5)$. For each gene the treatment effect $(\mathrm{TE})$ and its standard error (seTE) is plotted for each of the two Validation cohorts (1 and 2). Results of fixed and random effects meta-analysis for the log fold change (FC) of aspirin exposure for each gene and $95 \%$ confidence intervals (CI) are plotted.

Figure 3 Functional characterization of aspirin's effects on platelet gene expression. Panel A: Selected, top pathways to emerge from Gene Set Enrichment Analysis (GSEA, see Methods). For each selected pathway, its rank as well as direction of enrichment is listed. See Supplementary File 2 for full GSEA results. Panel B: Connectivity Map analyses (see Methods). A density plot of summary scores (tau, $\mathrm{x}$-axis) for different sets of small molecules in the Connectivity Map database: All small molecules $(\mathrm{n}=$ $2837)$ or subsets based on their mechanisms of action: "Cyclooxygenase inhibitors" $(\mathrm{n}=67)$, "Thromboxane synthase inhibitors" $(\mathrm{n}=13)$, "NFKB inhibitors" $(\mathrm{n}=35)$, and "AMPK activators" $(\mathrm{n}=4)$. The table lists those small molecules with the highest tau summary scores, their individual scores, and attributed mechanisms of action. Panel C: Fold change in platelet 18S ribosomal RNA (rRNA, y-axis) before (Visit 1) or after 4 - or 8 -weeks of $325 \mathrm{mg}$ /day aspirin therapy (Visits 2 and 3, respectively).

Figure 4 Aspirin's effect on platelet gene expression and association with non-COX1 dependent platelet function measures. Panel A: Effect of aspirin on an aggregate Aspirin Exposure Signature (AES, see Methods) that aggregates the effects of aspirin on the top aspirin-responsive genes into a single score. Values indicate p-value for comparisons between exposures. Panel B and C: The AES (x-axis) in each of the 
cohorts (Discovery, Validation 1, and Validation 2) is plotted against a previously described platelet function scores (PFS, y-axis) which is an aggregate a panel of individual non-COX1 dependent platelet function assays. Points represent individual patients, straight line represents best fit linear regression, shaded region represents $95 \%$ confidence intervals. Platelet function and AES scores were measured at baseline (Panel B) as well as after 4 weeks of aspirin exposure (Panel C). Panels D and E: Forest plots of effects (TE) and their standard errors (seTE) from linear regression models of AES vs. PFS in each cohort as well as results from fixed- and random-effects meta-analyses across cohorts. Results from baseline samples (Panel D) and on-aspirin samples (Panel E) are plotted.

Acknowledgements: None

Author Contributions:

Wrote Manuscript (RM, DV), Designed Research (DV, GSG, TLO, SSD), Performed Research (RM, DV, AW), Analyzed Data (RM, AW).

Supplementary File names and contents:

Supplementary File 1 - "AspirinDifferentialExpression.xlsx" - Provides the results of the effects of aspirin (either dose) on platelet gene expression for all genes that passed quality control (AspirinDifferentialExpression tab). Column header definitions the 'DataDictionary' tab.

Supplementary File 2 - "AspirinGSEA.xlsx" - Provides the full gene set enrichment analysis (GSEA) results for gene sets that were enriched for genes associated with aspirin exposure ('AspirinEnrichment' tab) or no drug exposure ('NoDrugEnrichment' tab). The 'DataDictionary' tab provides column definitions.
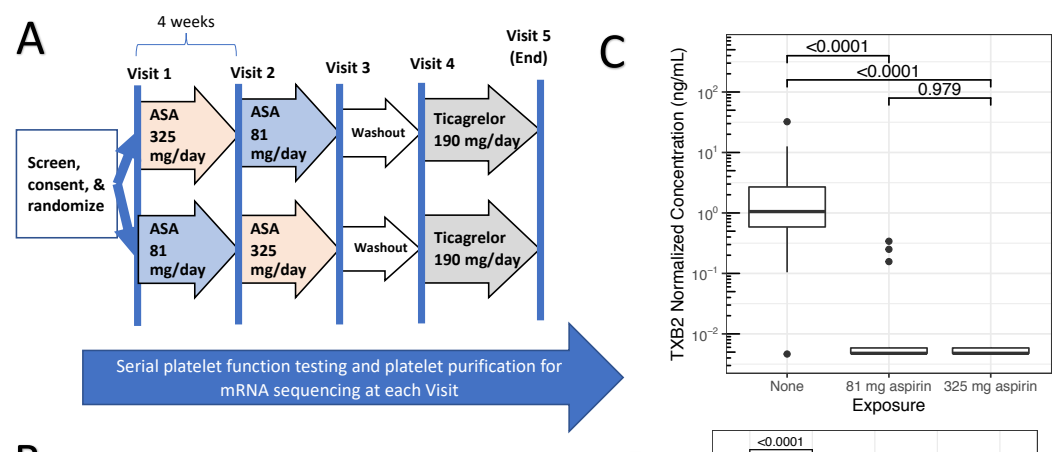

B

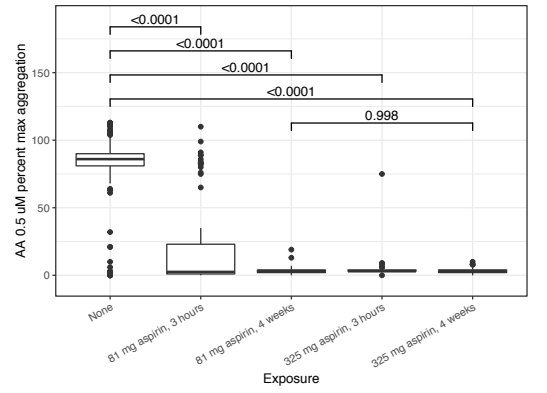

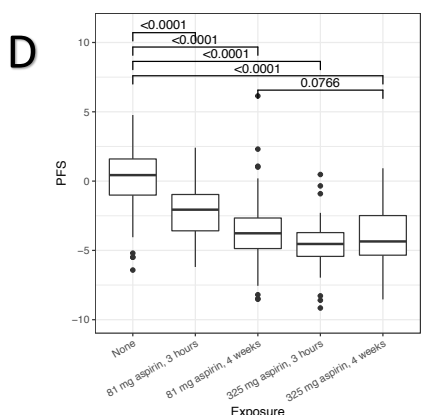



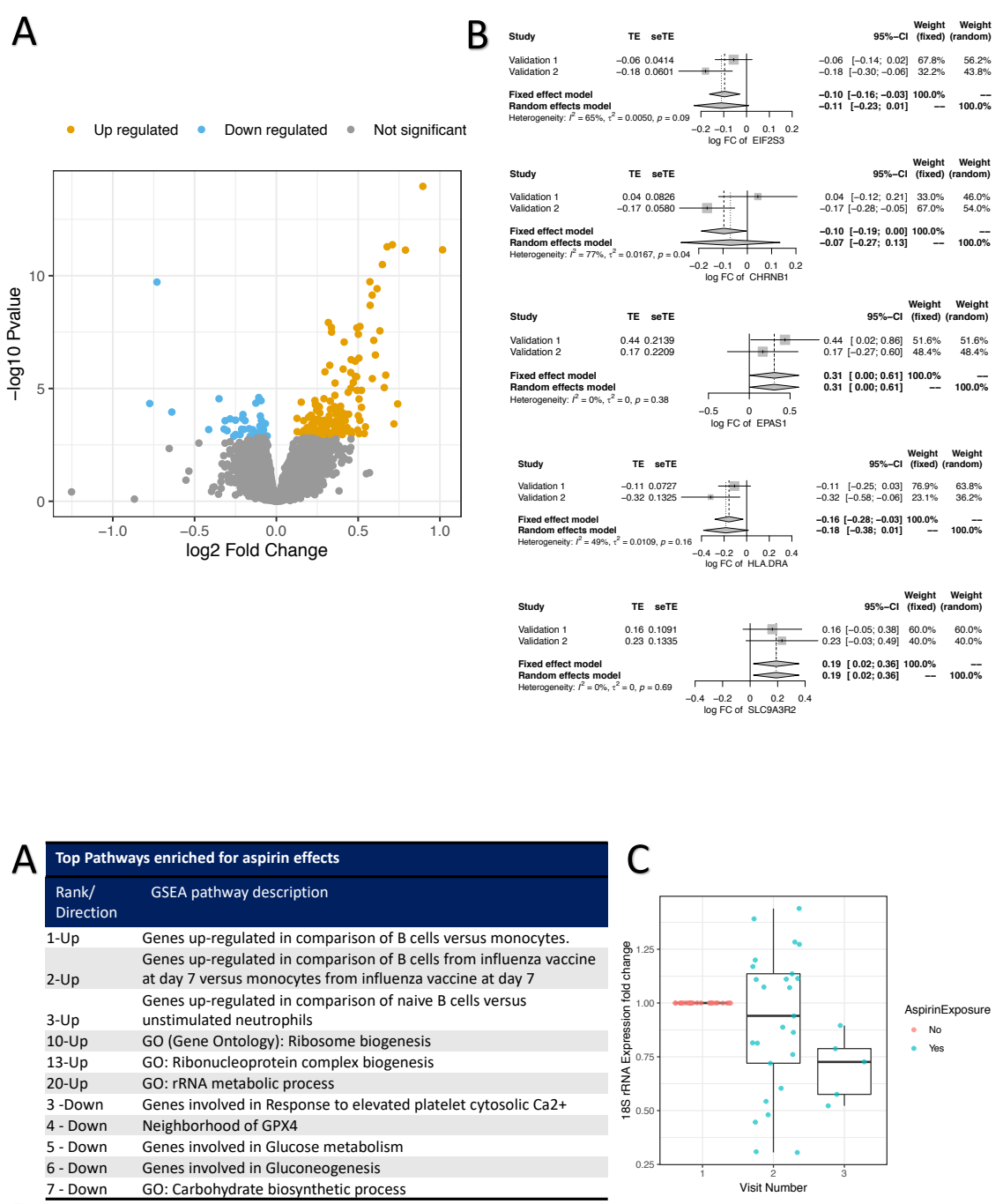

B

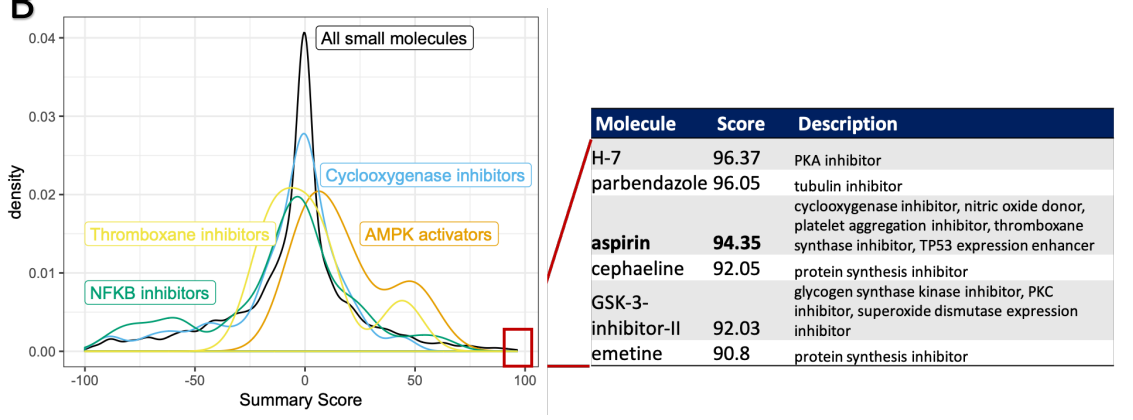



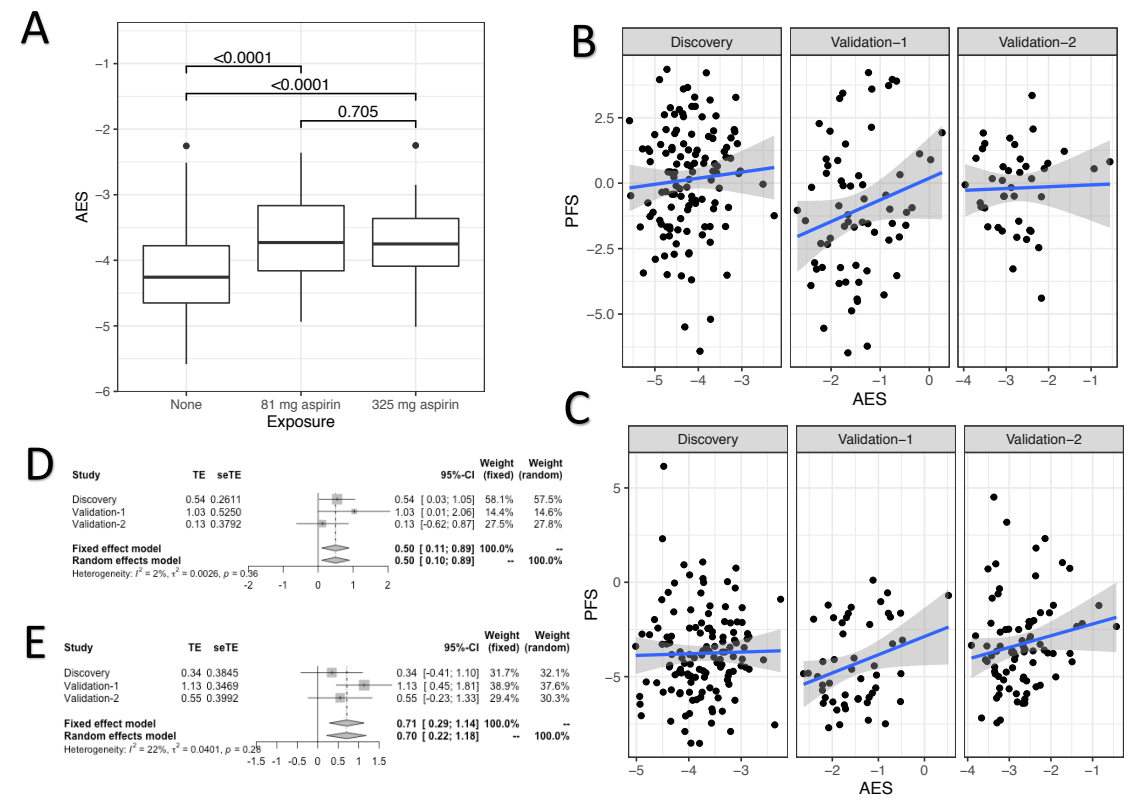